\title{
Nonlinear states and dynamics in a synthetic frequency dimension
}

\author{
Aleksandr K. Tusnin $\odot,{ }^{*}$ Alexey M. Tikan, ${ }^{\dagger}$ and Tobias J. Kippenberg ${ }^{\ddagger}$ \\ Institute of Physics, Swiss Federal Institute of Technology Lausanne (EPFL), Lausanne, Switzerland
}

(Received 12 May 2020; accepted 10 July 2020; published 13 August 2020)

\begin{abstract}
Recent advances in the study of synthetic dimensions revealed a possibility to employ the frequency space as an additional degree of freedom which allows for investigating and exploiting higher-dimensional phenomena in a priori low-dimensional systems. However, the influence of nonlinear effects on the synthetic frequency dimensions was studied only under significant restrictions. In the present paper, we develop a generalized meanfield model for the optical field envelope inside a single driven-dissipative resonator with quadratic and cubic nonlinearities, whose frequencies are coupled via an electro-optical resonant temporal modulation. The leadingorder equation takes the form of a driven Gross-Pitaevskii equation with a cosine potential. We numerically investigate the nonlinear dynamics in such a microring resonator with a synthetic frequency dimension in the regime where parametric frequency conversion occurs. We observe that the modulation brings additional control to the system, enabling one to readily create and manipulate bright and dark dissipative solitons inside the cavity. In the case of anomalous dispersion, we find that the presence of electro-optical mode coupling confines and stabilizes the chaotic modulation instability region. This leads to the appearance of an unconventional type of stable coherent structure which emerges in the synthetic space with restored translational symmetry, in a region of parameters where conventionally only chaotic modulation instability states exist. This structure appears in the center of the synthetic band and, therefore, is referred to as the band soliton. Finally, we extend our results to the case of multiple modulation frequencies with controllable relative phases creating synthetic lattices with nontrivial geometry. We show that an asymmetric synthetic band leads to the coexistence of chaotic and coherent states of the electromagnetic field inside the cavity, i.e., dynamics that can be interpreted as chimeralike states. Recently developed $\chi^{(2)}$ microresonators can open the way to experimentally exploring our findings.
\end{abstract}

DOI: 10.1103/PhysRevA.102.023518

\section{INTRODUCTION}

The idea of unification of physical theories by using higher-dimensional models beyond the usual space-time paradigm has arisen in the early years of development of quantum mechanics [1] and became an important precursor for modern unification theories [2]. However, investigation of effects presented in higher dimensions faces apparent challenges related to the number of dimensions provided by conventional physical systems. Boada et al. [3] have proposed to address these challenges by extending the well-established quantum simulator platform based on cold atoms with an additional synthetic dimension. The essence of the proposed idea was to encode an additional dimension into another degree of freedom (atomic spin state in this case) in the way that an effective Hamiltonian is analogous to a higher-dimensional one.

\footnotetext{
*aleksandr.tusnin@epfl.ch

†alexey.tikan@epfl.ch

†tobias.kippenberg@epfl.ch
}

Published by the American Physical Society under the terms of the Creative Commons Attribution 4.0 International license. Further distribution of this work must maintain attribution to the author(s) and the published article's title, journal citation, and DOI.
Since then, the concept of synthetic dimensions has been extended and used in various branches of physics [4]. It acquired special significance in photonics, where it provides a platform for exploring otherwise hardly accessible physical phenomena [5] and employment of synthetic dimensions allows for the dimensional extension employing only internal degrees of freedom of a system. This approach has been successfully applied to simulating particle random walk [6], effects of Bloch oscillations [7], unidirectional invisibility and unconventional reflection in parity-time symmetric systems [8], Anderson localization [9,10], etc. Recently, synthetic dimensions have been used in the studies of topological photonics [11-13]. Observation of a large variety of topological effects employing the synthetic frequency dimension has been proposed theoretically $[14,15]$ or realized experimentally [16-19].

Synthetic dimensions in photonics can be realized using different physical mechanisms [20]. For example, coupled oscillating waveguides [16], pair of coupled unequal loops [21], and phase modulation inside a ring cavity [22] allows for encoding a synthetic dimension into spatial discrete models, arrival time of pulses, and resonator modes, respectively. We will refer to the latter case as synthetic frequency dimensions. It can be created by inserting an electro-optical (EO) modulator into the ring resonator circumference [22-24]. Modulating an intracavity field at a frequency equal to an integer number of free spectral ranges (FSRs) [Fig. 1(a)], 

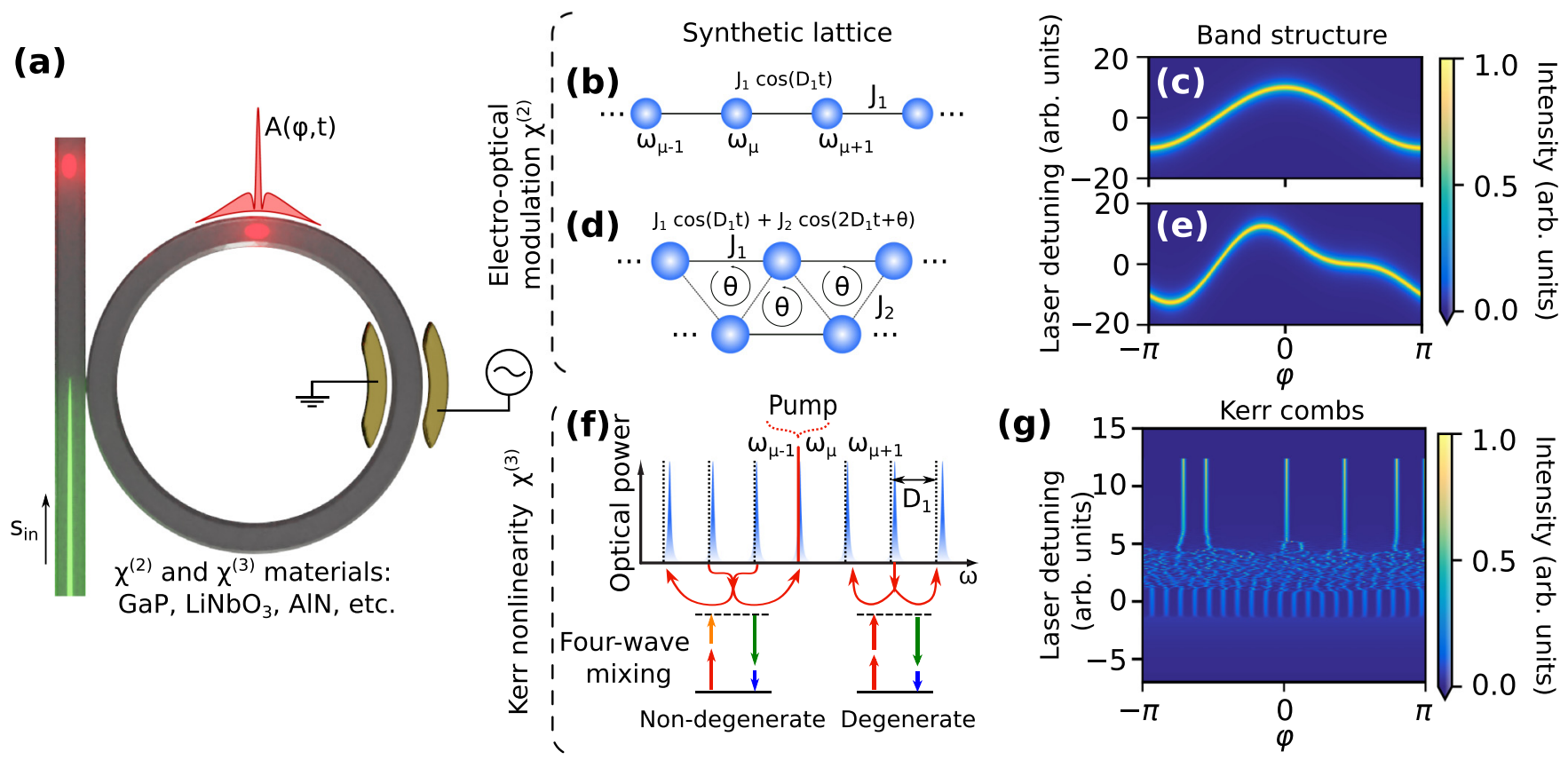

FIG. 1. Dynamically modulated optical cavity with $\chi^{(2)}$ and $\chi^{(3)}$ susceptibilities. (a) Optical cavity with an integrated EO (electro-optical) modulator. The modulation frequency is integer number of FSRs (free spectral ranges): $\Omega=s D_{1}$, where $s \in \mathbb{N}$. Due to the modulation, modes with frequencies $\omega_{\mu}$ and $\omega_{\mu+s}$ become coupled with coupling strength $J_{s}$ creating a synthetic lattice. $A(\varphi, t)$ represents a slowly varying envelope of the interactivity field in the rotating frame $\varphi=\phi-D_{1} t$, where $\phi$ is the polar angle. (b) Schematics of the lattice with the nearestneighbor coupling $(s=1)$. (c) Corresponding cavity field response with $J_{1}=10 \kappa / 2$, which represents the band structure. (d),(e) The same as (b),(c) but in the case of dual-tone modulation with relative phase $\theta=\pi / 2$ and $J_{2}=0.45 J_{1}$. (f) Displacement of the cavity resonance (in blue) from their exact equidistant positions (black dotted lines) due to the presence of dispersion. (g) Conventional nonlinear dynamics in a Kerr optical microresonator with anomalous group-velocity dispersion.

one can establish an effective photon flux between different optical modes supported by the resonator. In the case of the nearest-neighbor coupling (single FSR modulation) this system becomes similar to a one-dimensional chain of identical atoms [see Fig. 1(b)]. However, in contrast to solid state physics [25], the modulated cavity modes play the role of a direct space, whereas time acts as a reciprocal one. Hence, exciting a cavity with an external laser which operates at a frequency $\omega_{p}$ close to the resonant $\omega_{0}$ and measuring the intracavity field response as a function of detuning $\omega_{0}-\omega_{p}$, one can readily obtain a cosinelike band structure of the chain [26] [see Fig. 1(c)]. Furthermore, applying dual-tone modulation creates an effective two-dimensional frequency crystal [Fig. 1(d)] with controllable coupling strength (applied voltage) and phase flux (relative modulation phase) as introduced in the pioneering work by Dutt et al. [26]. Due to nonzero phase flux, the corresponding band structure has a nonreciprocal profile [Fig. 1(e)].

Strikingly, the role of nonlinearity in photonic synthetic frequency dimension is hardly explored; however, it is of particular importance for simulating locally interacting Hamiltonians [27-29] in complex many-body systems which are actively investigated in the context of photonic quantum simulator development [4]. Yuan et al. have proposed a scheme that employs Kerr nonlinearity to achieve the local interaction between the simulated particles [28]. They have simulated a synthetic state governing by an effective Bose-Hubbard Hamiltonian and successfully explored the photon blockade effect. Even though this approach requires fulfillment of very restrictive conditions (such as zero dispersion and conserved total number of photons, which restrains this study to lowpower regime), it is nonetheless very powerful since experimental platforms for realizing synthetic frequency dimensions often include materials with nonzero $\chi^{(3)}$ optical susceptibility.

Remarkably, balance between cubic nonlinearity and dispersion [see Fig. 1(f)] allows generating different types of solitary waves, including solitons [30,31]. In optical microcavities, an additional balance between parametric gain and cavity losses leads to the formation of dissipative Kerr solitons (DKS) [Fig. 1(g)]. Today, it is a very active field of research with wide raging applications [32-34]. Theoretically, dissipative solitons have been predicted in $\chi^{(2)}$ resonators as well $[35,36]$. Recent experimental observations with such photonic platforms as lithium niobate [37-39], aluminum nitride [40], and gallium phosphide [41] along with theoretical activities [42-45] in $\chi^{(2)}$ (and $\chi^{(2)}-\chi^{(3)}$ ) microresonators create a promising basis for the future development of this field and open opportunities for investigation of nonlinear topological photonics [17,46].

The present paper investigates the nonlinear dynamics in a dispersive cavity with $\chi^{(2)}$ and $\chi^{(3)}$ optical susceptibilities where voltage-induced phase modulation creates a synthetic frequency dimension. Starting from the coupled-mode formalism, we derive a mean-field Gross-Pitaevskii equation with a cosine potential which describes nonlinear dynamics of a resonantly modulated intracavity field in microresonators and fiber loop cavities [47]. We found that the modulation leads 
to predictable dissipative Kerr solitons (DKSs) [34,48] emergence and possibility of generation soliton crystals on demand [49-51]. We found that the modulation instability (MI) becomes bounded by the curved bistability region. Surprisingly, with increasing of the coupling rate, stable coherent structures emerge in the MI region, which we call band soliton. These states appear to be dispersionless, which makes them of particular interest in the context of synthetic frequency dimensions. Introducing a second tone to the intracavity phase modulation, we effectively create a nontrivial geometry which enables a nonreciprocal photon transfer [26]. This leads to the coexistence of stable coherent and chaotic regions which we interpret as chimeralike states [52]. Our results highlight the rich physics that can be accessed in synthetic dimensions with cubic nonlinearity.

\section{THEORY}

We consider an optical ring coupled to a bus waveguide with external coupling rate $\kappa_{\text {ex }}$ [Fig. 1(a)]. The cavity excited by a monochromatic laser with photon flux $s_{\text {in }}=\sqrt{P / \hbar \omega_{p}}$ ( $P$ is the input power) and frequency $\omega_{p}$, which is close to resonance frequency $\omega_{0}$. We suppose the modes being not equally spaced due to the dispersion, so the mode frequency $\left(\omega_{\mu}\right)$ depends on the mode number $(\mu)$ as $\omega_{\mu}=\omega_{0}+D_{1} \mu+$ $\mu^{2} D_{2} / 2$, where $D_{1} / 2 \pi$ equals FSR and $D_{2}$ characterizes the group-velocity dispersion (GVD) [Fig. 1(f)]. A synthetic frequency dimension is created by an EO modulator at one part of the ring with modulation frequency $\Omega=s D_{1}$ with $s \in$ $\mathbb{N}$ [20]. Considering on-resonance coupling, only the modes with frequencies $\omega_{\mu}$ and $\omega_{\mu \pm s}=\omega_{\mu} \pm s D_{1}+\frac{D_{2}}{2}\left(s^{2} \pm 2 \mu s\right)$ interact. The dispersion shifts the resonance positions, leading to altering of nearest-neighbors coupling efficiency. The linear equation of motion for the slowly varying mode amplitude $b_{\mu}$ can be written as

$$
\frac{\partial b_{\mu}}{\partial t}=\frac{i J_{s}}{2}\left(b_{\mu-s} e^{i \frac{D_{2} s}{2}(2 \mu-s) t-i \theta}+b_{\mu+s} e^{-i \frac{D_{2} s}{2}(2 \mu+s) t+i \theta}\right),
$$

where $J_{S}$ is the coupling rate with an arbitrary global phase $\theta$ corresponding to modulation $J \cos \left(s D_{1} t+\theta\right)$. By employing the Fourier transform of the field, one can deduce that this term may be rewritten as cosine potential for the electric field, so the governing equation for the electric-field envelope in $\chi^{(3)}$ resonator under EO modulation takes the form of the driven-damped Gross-Pitaevskii equation (GPE) (see Appendix A for the derivation). In dimensionless form, it can be written as

$$
\begin{aligned}
\frac{\partial \Psi}{\partial \tau}= & -\left(1+i \zeta_{0}\right) \Psi+i d_{2} \frac{\partial^{2} \Psi}{\partial \varphi^{2}}+i|\Psi|^{2} \Psi \\
& +i J \cos (s \varphi+\theta) \Psi+f,
\end{aligned}
$$

where normalized variables $\tau=t / \tau_{\mathrm{ph}}, \tau_{\mathrm{ph}}=2 / \kappa$ is the photon lifetime, $d_{2}=D_{2} / \kappa, \zeta_{0}=2 \delta \omega / \kappa, \delta \omega=\omega_{0}-\omega_{p}, J=$ $2 J_{s} / \kappa, f=\sqrt{8 \kappa_{e x} g_{0} / \kappa^{3}} s_{\text {in }}, \Psi=\sqrt{2 g_{0} / \kappa} A, \kappa=\kappa_{\text {ex }}+\kappa_{0}, \kappa_{0}$ is the intrinsic loss rate, $g_{0}$ is the single-photon Kerr frequency shift, $A$ describes the optical field envelope, and normalized such that $\int_{0}^{2 \pi}|A|^{2} d \varphi / 2 \pi$ is the number of photons inside the cavity. In the case of $J=0$ this equation corresponds to conventional LLE [33].
Let us begin with the analysis of stable solutions in the dispersionless limit $\left(d_{2}=0\right)$. Introducing $I=|\Psi|^{2}$, one can readily derive the cubic equation

$$
\left\{1+\left[I+J \cos (s \varphi)-\xi_{0}\right]^{2}\right\} I=f^{2} .
$$

The roots of this equation can be analyzed through its discriminant $\Delta$ (see Appendix B). Depending on the sign of $\Delta$, there are three scenarios for solutions of Eq. (3): if $\Delta<0$ there is one real root and two complex conjugated roots, if $\Delta=0$ roots are real and at least two of them are equal, and if $\Delta>0$ roots are real distinct numbers. Thus a negative (positive) discriminant corresponds to monostable (bistable) solutions, and in order to determine the bistability zone one needs to find $f^{2}$ and $\zeta_{0}$ such that the discriminant equals zero. Since Eq. (3) explicitly depends on $\varphi$, the discriminant becomes $\varphi$ dependent; therefore, different spatial parts of the cavity are found at different parts of the stability diagram at the same value of laser detuning [see Fig. 2(a)].

We note that a similar effect can be achieved imposing external resonant modulation on the pump laser [52,53]. External modulation has been employed for DKSs locking and manipulations creating an effective potential that traps DKSs [54]; it also helps to trigger platicon generation [55]. However, the $\varphi$ dependence lies in the right-hand side of Eq. (3). Therefore, it is expected that the internal phase modulation will provide an additional degree of freedom for controlling emergent coherent structures as well.

The threshold value $f^{2}$ which corresponds to the triple real root of Eq. (3) can be obtained analytically, and it equals $f_{\min }^{2}=8 \sqrt{3} / 9$, which coincides with the critical value for the resonance tilt for LLE [56]. Remarkably, this result does not depend on $\varphi$, despite the $\varphi$ dependence of Eq. (3).

\section{NUMERICAL SIMULATIONS}

\section{A. Dynamics of dissipative solitons and platicons}

For further analysis we consider the case $s=1$. In Fig. 2(a) we show the values of the discriminant $\Delta$ as a function of $\varphi$ and $\zeta_{0}$ for pump rate $f^{2}=6$ and coupling $J=3$. As one can see, the presence of the potential leads to bending of the bistable zone in a way that for a given detuning the system can be simultaneously on the monostable and bistable branches. With increasing of coupling strength $J$, the bistability zone bends further and goes deeper into the effectively red-detuned region $\left(\zeta_{0}>0\right)$ [see Fig. 2(a)].

We continue the further analysis by performing numerical simulation of GPE (2), taking $d_{2}= \pm 0.01$, and scanning the cavity from the blue- $\left(\zeta_{0}<0\right)$ to the red-detuned side. We employ numerical integration utilizing the split-step Fourier method [30]. The positive (negative) value of $d_{2}$ corresponds to anomalous (normal) dispersion regimes. We analyze these cases separately.

a. Anomalous dispersion. We observe that the presence of the potential in GPE (2) breaks the translational symmetry along the $\varphi$ coordinate and leads to confinement of the MI region [52]. We observe that chaotic patterns do not penetrate into bistable zones, and DKSs appear at the center of the cavity [Fig. 2(b)]. The latter might be qualitatively understood through the analysis of the steady-state dispersionless linear solution, which can be considered as a background for the 

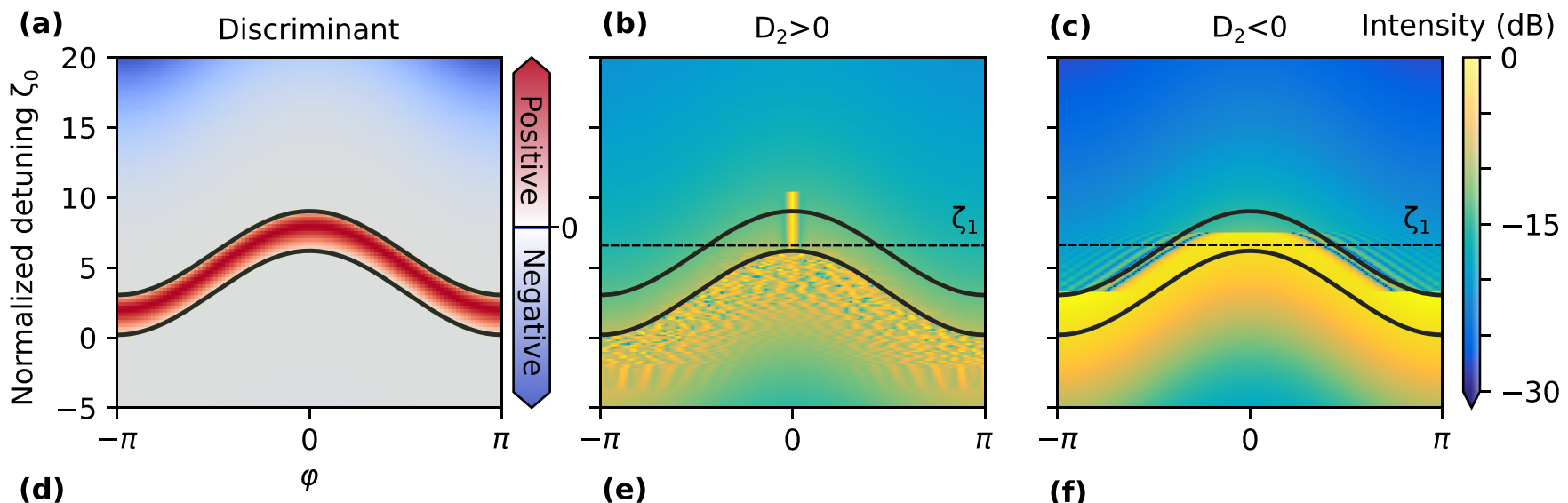

(e)
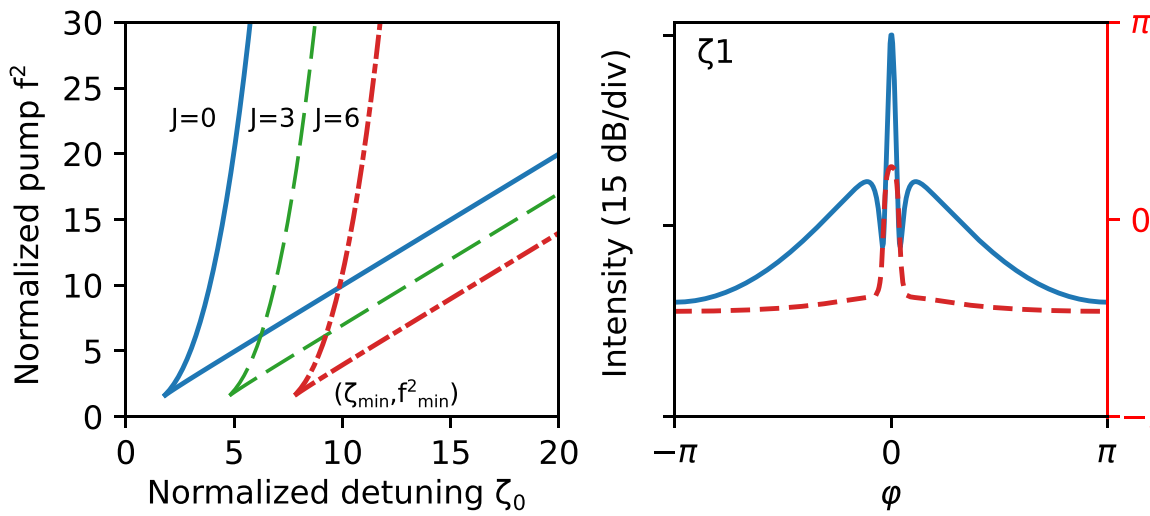

(f)

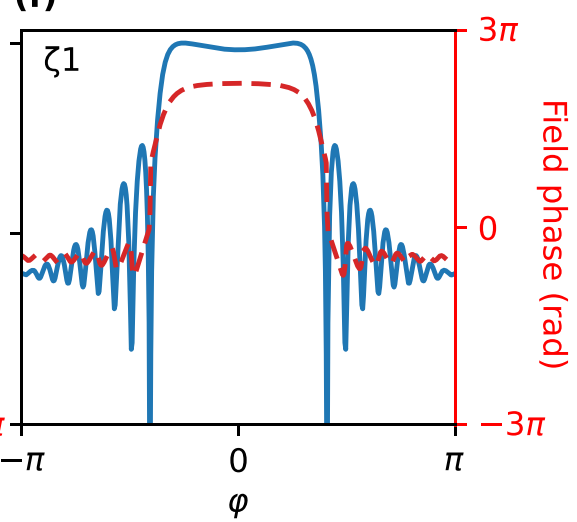

FIG. 2. Bistable branches and DKS (dissipative Kerr soliton)-platicon-existence range. (a) Value of the discriminant $\Delta$ [Eq. (B1)] for coupling rate $J=3$ and pump $f^{2}=6$ as a function of $\zeta_{0}$ and $\varphi$; (b),(c) intracavity field in the case of anomalous and normal GVD (groupvelocity dispersion), respectively. Black solid lines represent value $\Delta=0$ and indicate the bistable zone. MI (modulation instability) does not penetrate the bistable region [below lower black line on (b)]. Soliton existence range is almost covered by the bistable region at $\varphi=0$. (d) Bistability range at $\varphi=0$ for coupling values $J=0$ (solid), $J=3$ (dashed), and $J=6$ (dot-dashed). With increase of coupling $J$, the bistable zone shifts into the effectively red-detuned region $\left(\zeta_{0}>0\right)$ preserving its width. (e) Amplitude (solid blue) and phase (dashed red) profiles of DKS for detuning $\zeta_{1}=6.3$ [dashed lines on (b),(c)]. (f) The same in the case of normal dispersion.

dissipative nonlinear structures in the cavity. The intracavity field can be expressed as

$$
\Psi=\frac{f}{1+i\left[\zeta_{0}-J \cos (\varphi)\right]} .
$$

Depending on normalized detuning, the field intensity has one $\left(\zeta_{0}>J, \varphi_{0}=0\right)$ or two $\left(\zeta_{0}<J, \varphi_{ \pm}= \pm \arccos \xi_{0} / J\right)$ maxima. When the modulated background has only one peak, a single DKS can be formed on it. Numerical simulations show that the DKS appears on the peak of the modulated background in the bistable region [Fig. 2(b)] [57]. The width of this region as a function of $f^{2}$ and $J$ can be calculated analytically (see Appendix B for the details), and we present it on Fig. 2(d) for coupling rates $J=0,3,6$. Surprisingly, this zone simply shifts into the effectively red-detuned region linearly with $J$, and the critical detuning for $f_{\min }^{2}$ is

$$
\zeta_{\min }=\sqrt{3}+J .
$$

In order to calculate the soliton existence range, we employ the Lagrangian perturbative approach [58,59]. First of all, we introduce the change of variable $\Theta=1 / \sqrt{2 d_{2}} \varphi$ to Eq. (2). Thus the equation for the Lagrangian density can be written as follows:

$$
\begin{aligned}
\mathcal{L}= & \frac{i}{2}\left(\Psi^{*} \frac{\partial \Psi}{\partial \tau}-\Psi \frac{\partial \Psi^{*}}{\partial \tau}\right)-\frac{1}{2}\left|\frac{\partial \Psi}{\partial \Theta}\right|^{2} \\
& +\frac{1}{2}|\Psi|^{4}+\left[J \cos (\alpha \Theta)-\zeta_{0}\right]|\Psi|^{2},
\end{aligned}
$$

where $\alpha=2 d_{2}$. The dissipative function is introduced in the form

$$
\mathcal{R}=-i \Psi+i f .
$$

The Lagrangian $L=\int \mathcal{L} d \Theta$ obeys

$$
\frac{\partial L}{\partial q_{i}}-\frac{d}{d \tau} \frac{\partial L}{\partial \dot{q}_{i}}=\int\left(\mathcal{R} \frac{\partial \Psi^{*}}{\partial q_{i}}+\mathcal{R}^{*} \frac{\partial \Psi}{\partial q_{i}}\right) d \Theta .
$$

Using the ansatz of a stationary soliton $\Psi=B \operatorname{sech}(B \Theta) e^{i \xi_{0}}$ and considering $q_{1}=B$ and $q_{2}=\xi_{0}$, we get (see Appendix $C$ for more details)

$$
\begin{aligned}
& \frac{d B}{d \tau}=-2 B+\pi f \cos \xi_{0}, \\
& \frac{d \xi_{0}}{d \tau}=\frac{1}{2} B^{2}-\zeta_{0}+J \delta^{2} \frac{\cosh \delta}{\sinh \delta} \frac{1}{\sinh \delta},
\end{aligned}
$$


where we define $\delta=\alpha \pi / 2 B$. Considering $\alpha \ll 1$ and using Tailor expansion we obtain the stable solution

$$
\begin{aligned}
B^{2} & =2\left(\zeta_{0}-J\right), \\
\cos \xi_{0} & =\frac{2 B}{\pi f} .
\end{aligned}
$$

From the latter we obtain the analytical expression of the maximum detuning for stable soliton in the presence of nearest-neighbor coupling

$$
\zeta_{\max }=\pi^{2} f^{2} / 8+J
$$

This result generalizes the known expression for the soliton existence range to the single-tone EO modulated cavity. Similar to the bistable zone, the maximum detuning $\zeta_{\max }$ simply shifts by $J$.

Increasing the modulation frequency [i.e., increasing $s$ in Eq. (2)] leads to period multiplication of the modulated background and allows for creating soliton crystals [49,51] with $s$ equally spaced DKSs. Alternatively, applying several modulation signals and having control of the modulation phase, one can control positions and the number of DKS in the cavity, which enables soliton tweezing [57] and, as shown later, leads to nontrivial hybrid dynamics.

b. Normal dispersion. In the context of the conventional LLE with normal GVD $\left(d_{2}<0\right)$, the dark solitons (also called platicons) are hard to excite by simple laser tuning (soft excitation) for relatively small detunings and pump rates [56]. In order to create them, one needs to use additional methods, such as pumped modulation [55], or pumping in the avoided mode crossings $[60,61]$. In this context, EO modulation provides with an effective flux of photons from the pumped resonance to sidebands, making platicons accessible without additional perturbations. In Fig. 2(c) one can see platicon generation in the resonantly modulated cavity. In contrast to the DKS, the platicons appear only when one part of the cavity passes the whole bistable region; however, Fig. 2(d) can still indicate the approximate platicon existence range.

\section{B. Confined MI region}

Let us restrict our consideration for the case of anomalous $\operatorname{GVD}\left(d_{2}>0\right)$. In the conventional LLE formalism, in order to generate DKS via the soft excitation, one needs to scan the resonance through the MI region. In this region, coherent structures randomly appear and collide with each other, and may give birth to rogue waves [62]. However, due to the modulated background, the nonlinear structures appear and interact at different parts of the resonator differently. In order to investigate the role of coupling $J$, we explore spatiotemporal diagrams at a fixed pump rate, detuning and coupling rate, and its nonlinear dispersion relation (NDR), which is essentially the Fourier transform of the spatiotemporal diagram along two axes: time $(t)$ and space $(\varphi)$. This two-dimensional Fourier transform gives information about effective NDR and reveals insights about linear dispersive and nonlinear waves in the system [63]. For instance, a single DKS will be presented as a line in this diagram with a slope, which indicates its group velocity; a breather is similar to DKS, but its profile consists of equally spaced lines, which indicate its breathing oscillation in time; the linear waves, which obey the linear dispersion law, lie along the parabola. One may see all these features in Figs. 3(a)-3(f). First of all, we chose simulation parameters as in Fig. 2(b), but with fixed detuning $\zeta_{0}=1.3$. On the spatiotemporal diagram Fig. 3(a) one can see how nonlinear structures periodically arise and oscillate in the vicinity of background maxima $\varphi_{ \pm}$, propagate towards the maximum of the background phase at $\varphi \approx 0$ [red dashed line in Fig. 4(b)], and annihilate. There are several distinct structures on the corresponding NDR [Fig. 3(d)]: the periodic lines along the slow frequency axis with opposite slopes correspond to the colliding structures which locally have a conventional DKS (dissipative Kerr soliton) profile; the parabola corresponds to dispersive waves which are emitted by the breathing DKS on the background. With increase of the detuning these structures come closer, get smaller group velocity, and interact more chaotically while the field in the vicinity $\varphi= \pm \pi$ rests unperturbed. Thus we observe that for relatively small coupling rates the potential leads to confinement of the MI (modulation instability) state.

However, with increasing coupling strength $(J=6,9)$, we observe how these constantly interacting solitons are transformed into a stable dispersionless structure [Figs. 3(b), 3(c), $3(\mathrm{e})$, and 3(f)]. For coupling rate $J=6$, we observe that the field starts to periodically oscillate in time. The corresponding NDR consists of a ladder of lines, which signifies the appearance of a dispersionless breathing structure. Further increase of the coupling $(J=9)$ stabilizes this structure; it becomes coherent and dispersionless. In the following sections we further explore this unconventional state.

\section{Band soliton}

With increase of coupling strength $J$, we observe that the MI region is getting stabilized at a certain detuning range, and stable (i.e., coherent) nonlinear structures emerge. In a linear dispersionless case with the nearest-neighbor coupling $(s=1)$, the intracavity field response for different detunings represents a band structure of a one-dimensional synthetic crystal. However, the presence of FWM introduces global nonlinear coupling between the modes, the efficiency of which is given by the chromatic dispersion. The latter signifies that the eigenfunction basis (see Ref. [26]) is modified, and the intracavity field response can no longer be considered as a band structure.

Figure 4(a) demonstrates this difference: the deterministic dispersionless response (see upper right inset) transforms into a complex structure, which contains localized chaotic and stable states. However, the notion of band structure remains important even in the nonlinear regime $[64,65]$. We observe that there is a threshold value of $J$ for a given pump rate $f$ when the unconventional coherent structures appear. Comparing the nonlinear response [Fig. 4(a)] with the dispersionless linear one [Fig. 4(a), inset], we notice that these structures emerge in the center of the band structure; thus we call them band solitons. In analogy to solid-state physics, we can introduce the notion of synthetic Bloch waves (BW) [26], existing in the frequency space. Their group velocity reaches its maximum in the part of the band structure with the highest slope steepness. The latter signifies that the stable nonlinear states appear due to the interplay between FWM and linear BW. When 
(a)

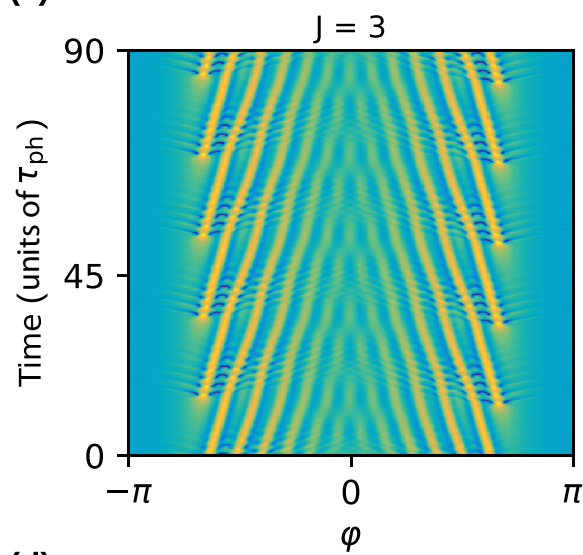

(d)

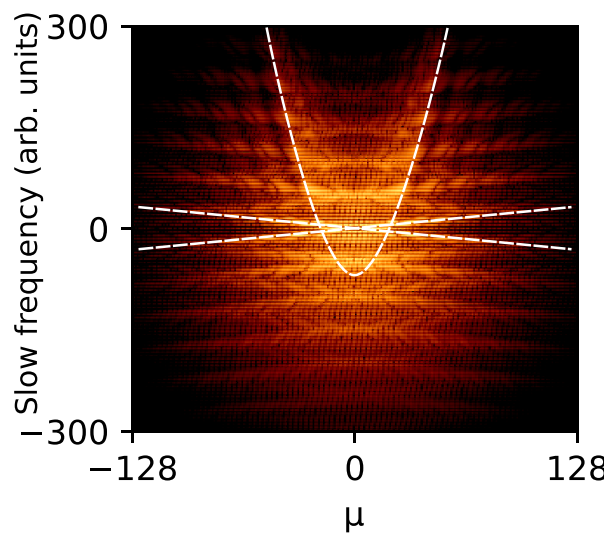

(b)

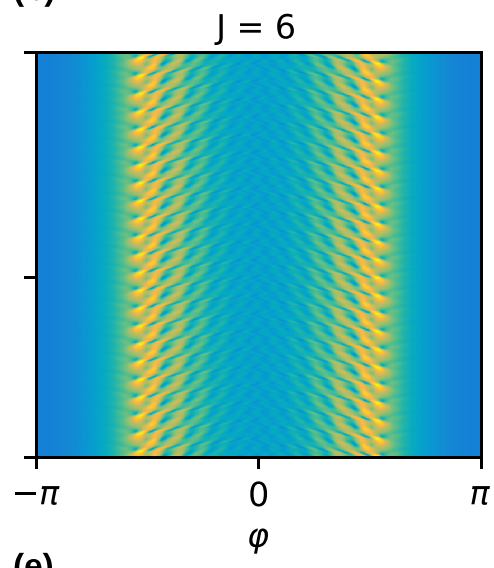

(e)

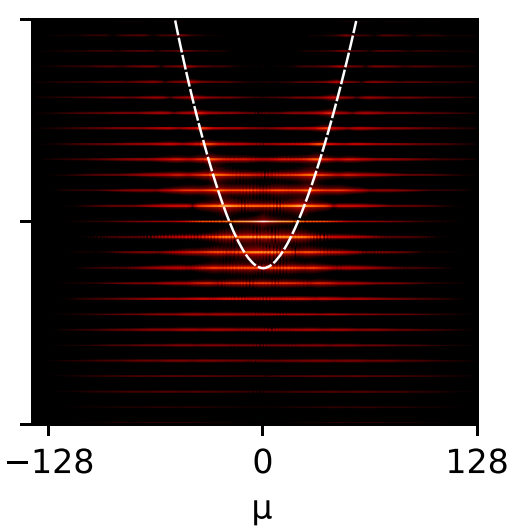

(c)

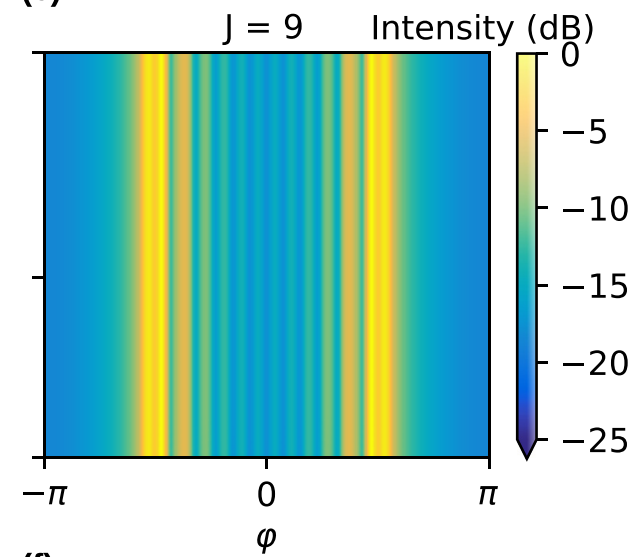

(f)

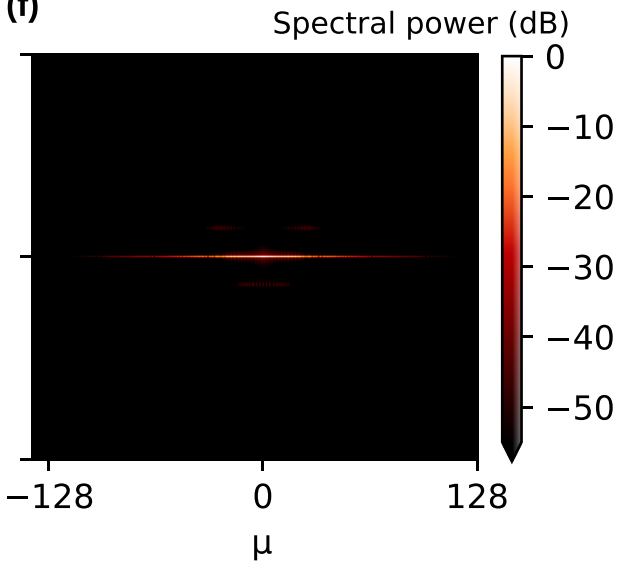

FIG. 3. Dynamics of the confined MI (modulation instability) region in the presence of potential. (a)-(c) Spatiotemporal diagrams of the intracavity field for $f^{2}=6, \zeta_{0}=1.28$. Coupling $J=3$ corresponds to (a), $J=6$ (b), and $J=9$ (c). (d)-(f) Corresponding NDR (nonlinear dispersion relation) which represents effective nonlinear dispersion relation of the system. One can notice how the dispersion relation transforms with increase of $J$. For $J=3$ the system consists of constantly appearing and colliding dispersionless structures [lines with opposite slopes in (d)], which radiate dispersive waves [parabola in (d)]; for $J=6$ (b),(e) the field oscillates as a whole and forms a ladder in the NDR profile, which indicates periodic breathing in time. Further increase of $J$ (c),(f) transforms the field into dispersionless stable dissipative structure.

the coupling strength is smaller than the threshold value, linear waves do not have sufficient velocity to redistribute perturbations induced by FWM. This regime corresponds to the confined MI. However, when the coupling strength exceeds the threshold value, the group velocity of the BW in the center of the band increases as well, and the BW can propagate faster along the frequency space and redistribute perturbations induced by FWM, leading to locking between the modes and the emergence of unconventional coherent states. This reasoning can also be applied to the explanation of the conventional DKS states' existence. As we have shown in previous sections, DKS appears exactly at the top of the band structure, where the group velocity of the BW equals zero; hence the photon flux from the pump is provided only due to FWM, and the synthetic BWs do not affect this process. Due to this fact, this soliton corresponds to the conventional soliton in optical $\chi^{(3)}$ microcavities.

Now we investigate the field's amplitude, phase, and spectrum at $\zeta_{\mathrm{I}}=4$ [Figs. 4(d) and 4(e)]. In the linear dispersionless case, the solution can be found analytically [Eq. (4)] and the field incorporates two maxima [Fig. 4(b)]. Corresponding spectral profile [Fig. 4(c), solid blue line] decays exponentially with mode number $\mu$, showing that the coupling rests the same for all the modes. Dispersion shifts the modes, decreasing coupling efficiency for higher-order modes and leading to truncation of the spectrum and emergence of a conventional EO comb [green dots on Figs. 4(c) and 4(e)] [37]. However, FWM shifts the resonances, enhancing coupling between the modes by restoring translational symmetry in the frequency space [see Fig. 4(e), solid blue line]. The spectrum of this state incorporates a flat part near the pump (at $-10 \mathrm{~dB}$ level) and decays slower than the EO comb, which signifies the restored coupling between the modes beyond the cutoff $[37,66,67]$.

In order to investigate stability of these states, we scan the cavity for different pump rates and coupling strengths. In Figs. 4(g) and 4(h) we present the phase diagram for single-tone modulation with coupling strengths $J=6$ and $J=9$, respectively, and compare it with the conventional LLE model [Fig. 4(f)]. The presence of the potential significantly changes the system dynamics; especially the MI region has different features. Band solitons emerge in a region around $\zeta_{0}=0$. With increasing $J$, their existence range increases along both axes. One can notice that this existence range 

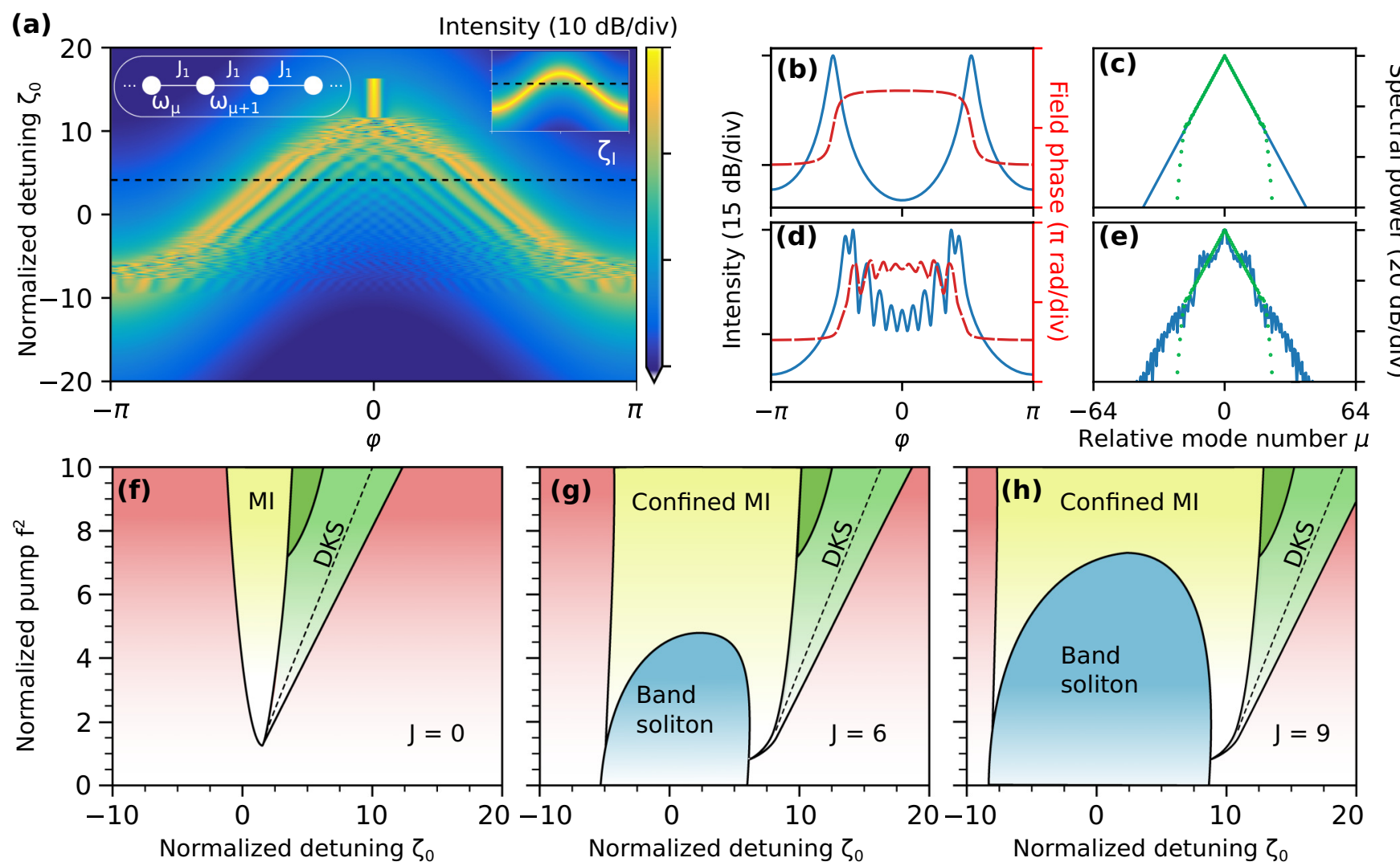

FIG. 4. New nonlinear states termed Band solitons for single-tone modulation. (a) Intracavity field for potential $J$ cos $\varphi$, coupling strength $J=9$, and pump rate $f^{2}=6$. Band solitons emerge in the detuning range $\zeta_{0} \in[-3.5,7]$. Insets show the linear dispersionless case: schematics of the lattice for coupling $J \cos \varphi$ in the linear case without dispersion (upper left) and corresponding cavity response (upper right). Horizontal line corresponds to detuning $\zeta_{\mathrm{I}}=4$, for which we examine field and spectrum profiles (b)-(e). (b) Linear field intensity (solid blue line) and phase (dashed red line). (c) Linear field spectrum without dispersion (solid blue line) and with dispersion $d_{2}=0.01$ (dotted green line). (d) Nonlinear field intensity (solid blue line) and phase (dashed red line). (e) Nonlinear field spectrum (solid blue line) and corresponding linear spectrum with dispersion (dotted green line). (f)-(h) Phase diagrams for coupling strengths $J=0, J=6$, and $J=9$. The red zone corresponds to continuous wave; the yellow zone indicates the confined MI (modulation instability) state; the green zone corresponds to the soliton existence range, which is predicted analytically. The dashed line indicates the end of the bistable region. The dark green region depicts DKS breathers. The blue zone indicates the existence range of band solitons, a type of dissipative coherent structure that appears in a conventional $(J=0)$ chaotic MI region.

is asymmetric, while in the linear case the band structure is symmetric [Fig. 4(a) upper right inset]. However, FWM induces self-phase modulation, leading to the frequency shift towards the effectively red-detuned zone, and the whole band obtains an offset from $\zeta_{0}=0$. The band solitons transform to conventional EO combs at the low pump rates when the FWM process becomes negligible. With increase of the pump rate, the band solitons start to breathe, become unstable, and transform to confined MI. Since the transition from the breathing state to the chaotic one is smooth, we joined these regions and labeled them as confined MI in Figs. 4(g) and 4(h) (note that we do not indicate here a narrow region of stable MI, which always manifests itself at negative values of detuning). This region appears to be wider than the MI region in the conventional LLE model [Fig. 4(f)].

\section{Chimeralike states}

Using two modulation frequencies and controlling the relative phase between them, one introduces a two-dimensional synthetic lattice [26] in the frequency space [Fig. 5(a), upper left inset]. The phase flux between the nodes can be controlled in this arrangement by the relative modulation phase. In particular, one can obtain asymmetric band structure introducing nonreciprocal frequency conversion [Fig. 5(a), upper right inset] [26]. We investigate nonlinear dynamics for a dual-tone modulation corresponding to the effective potential $J[\cos \varphi+0.45 \cos (2 \varphi+\theta)]$ with coupling $J=9$ and the relative phase $\theta=\pi / 2$. Nonreciprocal photon flow introduces a significant asymmetry in the corresponding spectral profile [Fig. 5(c)] [68]. However, in contrast to the single tone modulation, it is possible to find a region where one side of the band structure is almost flat, while another one has a maximum of its slope $\left[\zeta_{0} \approx 2\right.$ in Fig. 5(a)]. Therefore, for certain coupling rates fully chaotic dynamics manifests itself in a part of the cavity where the synthetic band structure slope (and hence the photon flow due to the linear BWs) is small, while another side can support a coherent band soliton existence. A similar intriguing feature was recently observed in systems with local coupling [52,69]. Following these 

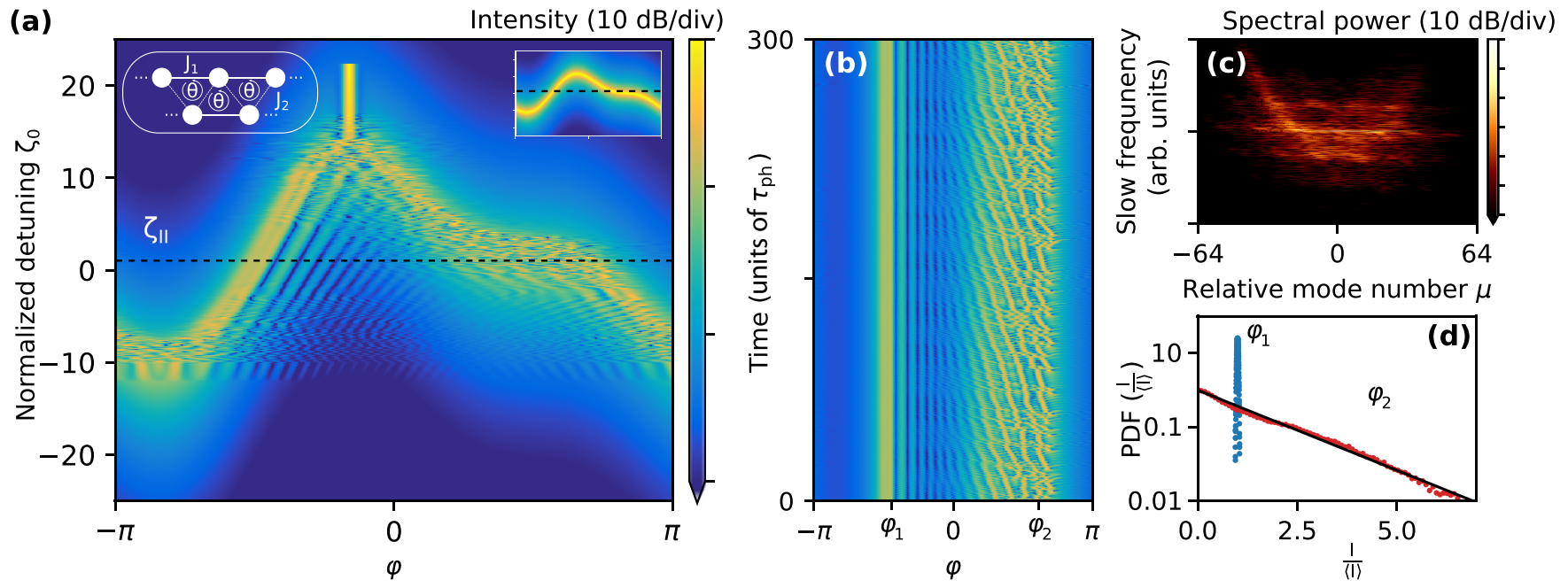

FIG. 5. Appearance of chimeralike states in the case of dual-tone modulation. (a) Intracavity field for potential $J[\cos \varphi+0.45 \cos (2 \varphi+$ $\theta$ )], coupling strength $J=9$ with relative phase $\theta=\pi / 2$ and $f^{2}=9$. Insets: corresponding schematics of lattice (upper left) in the linear case without dispersion and the cavity response (upper right). Horizontal line corresponds to detuning $\zeta_{\text {II }}=1.9$, for which we examine spatiotemproal diagram (b) and NDR (nonlinear dispersion relation) (c). (d) Single point PDF (probability density function) of the normalized intensity for two intracavity coordinates $\varphi_{1}=-1.4$ (blue) and $\varphi_{2}=1.8$ (red). Black solid line corresponds to exponential PDF exp $-\frac{I}{\langle I\rangle}$.

works, we refer to the observed phenomenon as a chimeralike state.

In order to investigate the chimeralike state, we extract the complex field envelope at the detuning value $\zeta_{I I}=1.9$ [black dashed line in Fig. 5(a)] and numerically propagate fixing all the parameters. The dynamics of the field modulus is shown in Fig. 5(b). Nonreciprocal photon transfer breaks the underlying symmetry of the system which also follows from the NDR [see Fig. 5(c)]. Computing the single point probability density function (PDF) of the intensity variation $I /\langle I\rangle(\langle I\rangle$ is averaged intensity in time) in coherent $\left(\varphi_{1}=-1.4\right)$ and incoherent $\left(\varphi_{2}=1.8\right)$ regions using $3 \times 10^{5}$ samples, we show that the $\mathrm{PDF}$ at $\varphi_{2}$ approaches the exponential (i.e., Gaussian distribution for the real part of the field), which can be considered as a signature of a fully developed MI stage [62] (also [70,71]), while at $\varphi_{1}$ it is close to deltalike distribution. Such states have no counterparts in DKS based on $\chi^{(3)}$ and single-tone driving.

\section{CONCLUSION}

In summary, we proposed a theoretical model which describes nonlinear dynamics of a modulated optical cavity with $\chi^{(2)}$ and $\chi^{(3)}$ optical susceptibilities and second-order GVD. We have shown that in the linear dispersionless limit the model describes the physics of a ring with a synthetic frequency dimension. Considering the dynamics of the full model, we found that despite the presence of GVD which breaks the translational symmetry there are coherent dispersionless structures for which the coupling remains resonant. There are two types of structures we have observed. First is found in the region of zero group velocity of the synthetic Bloch waves. They correspond to conventional DKS solutions of LLE but living on a modulated background. Applying different modulation signals, one can directly control the background modulation, hence control the number and positions of DKS, making soliton crystals and soliton tweezing readily accessible. The second type of structure is found at the maximum of the synthetic Bloch waves group velocity. Coherence of these structures, that we called band solitons, relies on the efficient photon transfer due to the linear mode coupling and Kerr nonlinearity which compensates the effect of dispersion. Therefore, such structures can be considered as nonlinear states in the synthetic frequency dimension. We generalized this result by including far neighbor coupling (double-tone modulation) into the model. We found that due to the nonreciprocal photon transfer the symmetry of the system is broken, which leads to the coexistence of stable coherent structures and chaos. We interpret these as the appearance of chimeralike states in the system.

We would like to emphasize that the proposed model can be used for further investigation of the synthetic frequency dimension as well as for simulations of EO combs in $\chi^{(2)}$ resonators. It can be readily generalized for an arbitrary dispersion profile, which can incorporate either higher-order dispersion $D_{3}, D_{4}$ or avoided mode crossings. Also, the potential of this model in the investigation of nonlinear effects in the synthetic frequency dimension in resonator lattices is of high interest. For example, by simulating a set of coupled GPE, one may explore the nonlinear dynamics of topological states, that can be created by changing the relative modulation phase of each ring.

As a physical platform for the model one can consider a high- $Q$ optical microcavity with $\chi^{(2)}$ and $\chi^{(3)}$ optical susceptibilities. With recent success in the fabrication process it has become possible to create optical cavities based on lithium niobate [37,38] or aluminum nitride [40], as well as gallium phosphide [41] photonic platforms, where the generation of Kerr [38,39] and EO modulation-based frequency combs [37] as well as Pockels soliton [40] has been reported. In particular, the scheme proposed in the current study can be easily realized on the $\mathrm{LiNbO}_{3}$ platform, where both FSR modulation [37] and self-starting Kerr combs [38] have been successfully demonstrated. 


\section{ACKNOWLEDGMENTS}

The authors thank J. Riemensberger, M. Karpov, and M. Churaev for fruitful discussions. This publication was supported by Contract No. D18AC00032 (DRINQS) from the Defense Advanced Research Projects Agency (DARPA), Defense Sciences Office (DSO). A.K.T. acknowledges support from the European Union's Horizon 2020 research and innovation programme under the Marie Skłodowska-Curie Grant Agreement No. 812818 (MICROCOMB).

\section{APPENDIX A: DERIVATION OF GROSS-PITAEVSKII EQUATION}

Let us consider a ring resonator with a phase modulator distributed at one part of the ring. If the modulator consists of $\chi^{(2)}$ active material, then it changes locally refractive index $n(\phi, t)$ and provides with linear coupling between different modes, which can be described by the equations of motion for the amplitudes $a_{\mu}$ as (see Supplemental Note 1 in Ref. [26])

$$
\frac{\partial a_{v}(t)}{\partial t}=-i \omega_{\nu} a_{\nu}(t)+i \sum_{\mu} J_{\mu-v}(t) a_{\mu}(t) .
$$

Let us suppose that the coupling coefficient does not depend on $\mu$ and depends harmonically on time as $J_{\mu-v}=$ $J_{s} \cos (\Omega t+\theta)$, where $\Omega$ is the modulation frequency and $\theta$ is the modulation phase. Under the transformation into rotating frame $\left(a_{v}=b_{\nu} e^{-i \omega_{\nu} t}\right)$, the equation reads

$$
\dot{b}_{\nu} e^{-i \omega_{\nu} t}=\frac{i}{2} \sum_{\mu} J_{s} b_{\mu} e^{-i \omega_{\mu} t}\left(e^{i \Omega t+i \theta}+e^{-i \Omega t-i \theta}\right)
$$

( $\dot{b}_{\mu}$ indicates time derivative). Supposing that we pump the resonator near to frequency $\omega_{0}$ such that

$$
\omega_{\mu}=\omega_{0}+\mu D_{1}+D_{2} \frac{\mu^{2}}{2}
$$

and modulation frequency $\Omega$ is chosen as $\Omega=s D_{1}$, where $s$ is an integer, the RHS of Eq. (A2) incorporates two exponentials:

$$
\begin{aligned}
& \omega_{\mu}+s D_{1}-\omega_{v}=D_{1}(\mu+s-v)+\frac{D_{2}}{2}\left(\mu^{2}-v^{2}\right), \\
& \omega_{\mu}-s D_{1}-\omega_{v}=D_{1}(\mu-s-v)+\frac{D_{2}}{2}\left(\mu^{2}-v^{2}\right) .
\end{aligned}
$$

Therefore, the resonant interaction appears between modes $\mu=v \pm s$, and Eq. (A2) simplifies to

$$
\dot{b}_{v}=\frac{i J_{s}}{2}\left(e^{i \theta} b_{v+s} e^{-i \frac{D_{2} s}{2}(2 v+s) t}+e^{-i \theta} b_{v-s} e^{i \frac{D_{2} s}{2}(2 v-s) t}\right) .
$$

Now we aim to find the corresponding equation of the cavity field. In optical cavity the field envelope may be presented as a Fourier series [48]

$$
A(\phi, t)=\sum_{\mu} a_{\mu} e^{i \mu \phi}=\sum_{\mu} b_{\mu} e^{i\left(\mu \phi-\omega_{\mu} t\right)} .
$$

Taking the time derivative, one obtains

$$
\dot{A}=\sum_{\mu}\left(\dot{b}_{\mu}-i \omega_{\mu} b_{\mu}\right) e^{i\left(\mu \phi-\omega_{\mu} t\right)} .
$$

Let us consider only the first term. Substituting Eq. (A6) yields

$$
\begin{aligned}
\sum_{\mu} \dot{b}_{\mu} e^{i\left(\mu \phi-\omega_{\mu} t\right)}= & i \sum_{\mu} e^{i\left(\mu \phi-\omega_{\mu} t\right)} J_{s}\left(a_{\mu-s} e^{i \frac{D_{2} s}{2}(2 \mu-s) t+i \theta}\right. \\
& \left.+a_{\mu+s} e^{-i \frac{D_{2} s}{2}(2 \mu+s) t-i \theta}\right)
\end{aligned}
$$

One may readily rearrange the exponential relations

$$
\begin{aligned}
& \omega_{\mu-s}=\omega_{\mu}-s D_{1}+\frac{D_{2}}{2}\left(s^{2}-2 \mu s\right), \\
& \omega_{\mu+s}=\omega_{\mu}+s D_{1}+\frac{D_{2}}{2}\left(s^{2}+2 \mu s\right),
\end{aligned}
$$

and the summation yields that modulation creates a potential for the electric field

$$
J_{s} \cos \left(\phi s-s D_{1} t+\theta\right) .
$$

Therefore, in the frame $\varphi$ rotating with speed $D_{1}$ such that $\varphi=\phi-D_{1} t$, the electric field obeys the following equation:

$$
\dot{A}=i J_{s} \cos (s \varphi+\theta) A \text {. }
$$

This result might be combined with Lugiato-Lefever formalism for Kerr combs in optical cavities [72], and hence the equation which governs the electric field in the presence of external pump with frequency $\omega_{p}=\omega_{0}+\delta \omega$ is

$$
\begin{aligned}
\frac{\partial A}{\partial t}= & -\left(\frac{\kappa}{2}+i \delta \omega\right) A+\frac{i D_{2}}{2} \frac{\partial^{2} A}{\partial \varphi^{2}}+2 i J_{s} \cos (s \varphi+\theta) A \\
& +i g_{0}|A|^{2} A+\sqrt{\kappa_{\mathrm{ex}}} s_{\mathrm{in}} .
\end{aligned}
$$

\section{APPENDIX B: STABLE DISPERSIONLESS LIMIT}

Since the cubic equation (3) is written for real value $|\Psi|^{2}$, the solution has to be real as well. However, it is well known that a cubic equation always possesses three roots, and they are characterized through its discriminant $\Delta$. In our case, the discriminant has the following form:

$$
\begin{aligned}
\Delta= & -27 f^{4}-4\left(1+\xi_{0}^{2}\right)^{2}+4 f^{2} \xi_{0}\left(9+\xi_{0}^{2}\right)+4 J \cos (s \varphi) \\
& \times\left(-3 f^{2}\left(3+\xi_{0}^{2}\right)+4 \xi_{0}\left(1+\xi_{0}^{2}\right)-J \cos (s \varphi)\{2\right. \\
& \left.\left.-3 f^{2} \xi_{0}+6 \xi_{0}^{2}+J \cos (s \varphi)\left[f^{2}-4 \xi_{0}^{2}+J \cos (s \varphi)\right]\right\}\right) .
\end{aligned}
$$

Solving the equation $\Delta=0$, we find values $f^{2}$ and $\zeta_{0}$ which determine the bistable zone.

\section{APPENDIX C: LAGRANGIAN PERTURBATIVE APPROACH}

In order to calculate the maximum detuning for DKS in a modulated cavity one needs to use the ansatz of a stationary soliton $\Psi=B e^{i \xi_{0}} \operatorname{sech}(B \Theta)$ in the Lagrangian density (6). Integrating it over $\Theta$ on the interval $(-\infty,+\infty)$ (under the assumption $D_{2} / \kappa \ll 1$ ), one gets the Lagrangian in the form

$$
L=-2 B \frac{\partial \xi_{0}}{\partial \tau}+\frac{1}{3} B^{3}-2 B \xi_{0}+\frac{J \alpha \pi}{\sinh \left(\frac{\alpha \pi}{2 B}\right)} .
$$

The right-hand side of Eq. (8) is not affected by the presence of the potential and coincides with works in [58,59]. 
[1] O. Klein, Quantum theory and five-dimensional relativity theory, in The Oskar Klein Memorial Lectures: Vol 1: Lectures by $C N$ Yang and $S$ Weinberg (World Scientific, Singapore, 1991), pp. $67-80$.

[2] P. S. Wesson, Five-dimensional Physics: Classical and Quantum Consequences of Kaluza-Klein Cosmology (World Scientific, Singapore, 2006).

[3] O. Boada, A. Celi, J. I. Latorre, and M. Lewenstein, Quantum Simulation of an Extra Dimension, Phys. Rev. Lett. 108, 133001 (2012).

[4] T. Ozawa and H. M. Price, Topological quantum matter in synthetic dimensions, Nat. Rev. Phys. 1, 349 (2019).

[5] A. Aspuru-Guzik and P. Walther, Photonic quantum simulators, Nat. Phys. 8, 285 (2012).

[6] A. Regensburger, C. Bersch, B. Hinrichs, G. Onishchukov, A. Schreiber, C. Silberhorn, and U. Peschel, Photon Propagation in a Discrete Fiber Network: An Interplay of Coherence and Losses, Phys. Rev. Lett. 107, 233902 (2011).

[7] M. Wimmer, M.-A. Miri, D. Christodoulides, and U. Peschel, Observation of Bloch oscillations in complex PT-symmetric photonic lattices, Sci. Rep. 5, 17760 (2015).

[8] A. Regensburger, C. Bersch, M.-A. Miri, G. Onishchukov, D. N. Christodoulides, and U. Peschel, Parity-time synthetic photonic lattices, Nature (London) 488, 167 (2012).

[9] I. D. Vatnik, A. Tikan, G. Onishchukov, D. V. Churkin, and A. A. Sukhorukov, Anderson localization in synthetic photonic lattices, Sci. Rep. 7, 4301 (2017).

[10] A. V. Pankov, I. D. Vatnik, D. V. Churkin, and S. A. Derevyanko, Anderson localization in synthetic photonic lattice with random coupling, Opt. Express 27, 4424 (2019).

[11] L. Lu, J. D. Joannopoulos, and M. Soljačić, Topological photonics, Nat. Photon. 8, 821 (2014).

[12] A. B. Khanikaev and G. Shvets, Two-dimensional topological photonics, Nat. Photon. 11, 763 (2017).

[13] T. Ozawa, H. M. Price, A. Amo, N. Goldman, M. Hafezi, L. Lu, M. C. Rechtsman, D. Schuster, J. Simon, O. Zilberberg, and I. Carusotto, Topological photonics, Rev. Mod. Phys. 91, 015006 (2019).

[14] Q. Lin, M. Xiao, L. Yuan, and S. Fan, Photonic Weyl point in a two-dimensional resonator lattice with a synthetic frequency dimension, Nat. Commun. 7, 13731 (2016).

[15] A. Dutt, M. Minkov, I. A. D. Williamson, and S. Fan, Higherorder topological insulators in synthetic dimensions, Light Sci. Appl. 9, 131 (2020).

[16] E. Lustig, S. Weimann, Y. Plotnik, Y. Lumer, M. A. Bandres, A. Szameit, and M. Segev, Photonic topological insulator in synthetic dimensions, Nature (London) 567, 356 (2019).

[17] Z. Yang, E. Lustig, G. Harari, Y. Plotnik, Y. Lumer, M. A. Bandres, and M. Segev, Mode-Locked Topological Insulator Laser Utilizing Synthetic Dimensions, Phys. Rev. X 10, 011059 (2020).

[18] W. Zhang and X. Zhang, Photonic quadrupole topological phases in zero-dimensional cavity with synthetic dimensions, arXiv:1906.02967.

[19] A. Dutt, Q. Lin, L. Yuan, M. Minkov, M. Xiao, and S. Fan, A single photonic cavity with two independent physical synthetic dimensions, Science 367, 59 (2020).

[20] L. Yuan, Q. Lin, M. Xiao, and S. Fan, Synthetic dimension in photonics, Optica 5, 1396 (2018).
[21] A. Schreiber, K. N. Cassemiro, V. Potoček, A. Gábris, P. J. Mosley, E. Andersson, I. Jex, and C. Silberhorn, Photons Walking the Line: a Quantum Walk with Adjustable Coin Operations, Phys. Rev. Lett. 104, 050502 (2010).

[22] T. Ozawa, H. M. Price, N. Goldman, O. Zilberberg, and I. Carusotto, Synthetic dimensions in integrated photonics: From optical isolation to four-dimensional quantum Hall physics, Phys. Rev. A 93, 043827 (2016).

[23] L. Yuan, Y. Shi, and S. Fan, Photonic gauge potential in a system with a synthetic frequency dimension, Opt. Lett. 41, 741 (2016).

[24] L. Yuan and S. Fan, Bloch oscillation and unidirectional translation of frequency in a dynamically modulated ring resonator, Optica 3, 1014 (2016).

[25] N. W. Ashcroft and N. D. Mermin, Solid State Physics (Brooks/Cole, South Melbourne, 1976).

[26] A. Dutt, M. Minkov, Q. Lin, L. Yuan, D. A. B. Miller, and S. Fan, Experimental band structure spectroscopy along a synthetic dimension, Nat. Commun. 10, 3122 (2019).

[27] T. Ozawa and I. Carusotto, Synthetic Dimensions with Magnetic Fields and Local Interactions in Photonic Lattices, Phys. Rev. Let. 118, 013601 (2017).

[28] L. Yuan, A. Dutt, M. Qin, S. Fan, and X. Chen, Creating locally interacting Hamiltonians in the synthetic frequency dimension for photons, arXiv:1909.12466.

[29] L. Barbiero, L. Chomaz, S. Nascimbene, and N. Goldman, Bose-Hubbard physics in synthetic dimensions from interaction Trotterization, arXiv:1907.10555.

[30] G. P. Agrawal, Nonlinear Fiber Optics (Springer, Berlin, 2000).

[31] Y. Kivshar and C. Agrawal, Optical Solitons (Elsevier Science, Amsterdam, 2003).

[32] L. A. Lugiato, F. Prati, M. L. Gorodetsky, and T. J. Kippenberg, From the Lugiato-Lefever equation to microresonator-based soliton Kerr frequency combs, Philos. Trans. R. Soc. A: Math., Phys. Eng. Sci. 376, 20180113 (2018).

[33] L. A. Lugiato and R. Lefever, Spatial Dissipative Structures in Passive Optical Systems, Phys. Rev. Lett. 58, 2209 (1987).

[34] T. J. Kippenberg, A. L. Gaeta, M. Lipson, and M. L. Gorodetsky, Dissipative Kerr solitons in optical microresonators, Science 361, eaan8083 (2018).

[35] A. V. Buryak, P. Di Trapani, D. V. Skryabin, and S. Trillo, Optical solitons due to quadratic nonlinearities: From basic physics to futuristics applications, Phys. Rep. 370, 63 (2002).

[36] F. Leo, T. Hansson, I. Ricciardi, M. De Rosa, S. Coen, S. Wabnitz, and M. Erkintalo, Frequency-comb formation in doubly resonant second-harmonic generation, Phys. Rev. A 93, 043831 (2016).

[37] M. Zhang, B. Buscaino, C. Wang, A. Shams-Ansari, C. Reimer, R. Zhu, J. M. Kahn, and M. Lončar, Broadband electro-optic frequency comb generation in a lithium niobate microring resonator, Nature (London) 568, 373 (2019).

[38] Y. He, Q.-F. Yang, J. Ling, R. Luo, H. Liang, M. Li, B. Shen, H. Wang, K. Vahala, and Q. Lin, Self-starting bi-chromatic $\mathrm{LiNbO}_{3}$ microcomb, Optica 6, 1138 (2019).

[39] Z. Gong, X. Liu, Y. Xu, M. Xu, J. B. Surya, J. Lu, A. Bruch, C. Zou, and H. X. Tang, Soliton microcomb generation at $2 \mu \mathrm{m}$ in z-cut lithium niobate microring resonators, Opt. Lett. 44, 3182 (2019).

[40] A. W. Bruch, X. Liu, Z. Gong, J. B. Surya, M. Li, C.-L. Zou, and H. X. Tang, Pockels soliton microcomb, arXiv:2004.07708. 
[41] D. J. Wilson, K. Schneider, S. Hönl, M. Anderson, Y. Baumgartner, L. Czornomaz, T. J. Kippenberg, and P. Seidler, Integrated gallium phosphide nonlinear photonics, Nat. Photon. 14, 57 (2020).

[42] W. R. Rowe, D. V. Skryabin, and A. V. Gorbach, Temporal quadratic solitons and their interaction with dispersive waves in lithium niobate nanowaveguides, Phys. Rev. Research 1, 033146 (2019).

[43] E. Podivilov, S. Smirnov, I. Breunig, and B. Sturman, Nonlinear solutions for $\chi^{(2)}$ frequency combs in optical microresonators, Phys. Rev. A 101, 023815 (2020).

[44] C. Mas Arabí, P. Parra-Rivas, C. Ciret, S. P. Gorza, and F. Leo, Modeling of quasi-phase-matched cavity-enhanced secondharmonic generation, Phys. Rev. A 101, 043818 (2020).

[45] V. E. Lobanov, N. M. Kondratiev, A. E. Shitikov, and I. A. Bilenko, Two-color flat-top solitonic pulses in $\chi^{(2)}$ optical microresonators via second-harmonic generation, Phys. Rev. A 101, 013831 (2020).

[46] D. Smirnova, D. Leykam, Y. Chong, and Y. Kivshar, Nonlinear topological photonics, Appl. Phys. Rev. 7, 021306 (2020).

[47] W. Stephan, Suppression of soliton interactions by phase modulation, Electron. Lett. 29, 31 (1993).

[48] T. Herr, V. Brasch, J. D. Jost, C. Y. Wang, N. M. Kondratiev, M. L. Gorodetsky, and T. J. Kippenberg, Temporal solitons in optical microresonators, Nat. Photon. 8, 145 (2014).

[49] D. C. Cole, E. S. Lamb, P. Del'Haye, S. A. Diddams, and S. B. Papp, Soliton crystals in kerr resonators, Nat. Photon. 11, 671 (2017).

[50] Y. He, J. Ling, M. Li, and Q. Lin, Perfect soliton crystals on demand, arXiv:1910.00114.

[51] M. Karpov, M. H. Pfeiffer, H. Guo, W. Weng, J. Liu, and T. J. Kippenberg, Dynamics of soliton crystals in optical microresonators, Nat. Phys. 15, 1071 (2019).

[52] A. U. Nielsen, Y. Xu, M. Ferré, M. G. Clerc, S. Coen, S. G. Murdoch, and M. Erkintalo, Engineered discreteness enables observation and control of chimera-like states in a system with local coupling, arXiv:1910.11329.

[53] M. H. Anderson, R. Bouchand, J. Liu, W. Weng, E. Obrzud, T. Herr, and T. J. Kippenberg, Photonic chip-based resonant supercontinuum, arXiv:1909.00022.

[54] E. Obrzud, S. Lecomte, and T. Herr, Temporal solitons in microresonators driven by optical pulses, Nat. Photon. 11, 600 (2017).

[55] V. Lobanov, G. Lihachev, T. J. Kippenberg, and M. Gorodetsky, Frequency combs and platicons in optical microresonators with normal gvd, Opt. Express 23, 7713 (2015).

[56] C. Godey, I. V. Balakireva, A. Coillet, and Y. K. Chembo, Stability analysis of the spatiotemporal Lugiato-Lefever model for Kerr optical frequency combs in the anomalous and normal dispersion regimes, Phys. Rev. A 89, 063814 (2014).

[57] J. K. Jang, M. Erkintalo, S. Coen, and S. G. Murdoch, Temporal tweezing of light through the trapping and manipulation of temporal cavity solitons, Nat. Commun. 6, 1 (2015).

[58] P. Grelu, Nonlinear Optical Cavity Dynamics: From Microresonators to Fiber Lasers (John Wiley \& Sons, New York, 2015).

[59] S. Wabnitz, Suppression of interactions in a phase-locked soliton optical memory, Opt. Lett. 18, 601 (1993).

[60] X. Xue, Y. Xuan, Y. Liu, P. H. Wang, S. Chen, J. Wang, D. E. Leaird, M. Qi, and A. M. Weiner, Mode-locked dark pulse Kerr combs in normal-dispersion microresonators, Nat. Photon. 9, 594 (2015)

[61] B. Y. Kim, Y. Okawachi, J. K. Jang, M. Yu, X. Ji, Y. Zhao, C. Joshi, M. Lipson, and A. L. Gaeta, Turn-key, high-efficiency Kerr comb source, Opt. Lett. 44, 4475 (2019).

[62] S. Coulibaly, M. Taki, A. Bendahmane, G. Millot, B. Kibler, and M. G. Clerc, Turbulence-Induced Rogue Waves in Kerr Resonators, Phys. Rev. X 9, 011054 (2019).

[63] K. P. Leisman, D. Zhou, J. W. Banks, G. Kovačič, and D. Cai, Effective dispersion in the focusing nonlinear Schrödinger equation, Phys. Rev. E 100, 022215 (2019).

[64] Y. Lumer, M. C. Rechtsman, Y. Plotnik, and M. Segev, Instability of bosonic topological edge states in the presence of interactions, Phys. Rev. A 94, 021801(R) (2016).

[65] D. D. Solnyshkov, O. Bleu, B. Teklu, and G. Malpuech, Chirality of Topological Gap Solitons in Bosonic Dimer Chains, Phys. Rev. Lett. 118, 023901 (2017).

[66] K. P. Ho and J. M. Kahn, Optical frequency comb generator using phase modulation in amplified circulating loop, IEEE Photon. Technol. Lett. 5, 721 (1993).

[67] M. Kourogi, N. Ken'ichi, and M. Ohtsu, Wide-span optical frequency comb generator for accurate optical frequency difference measurement, IEEE J. Quantum Electron. 29, 2693 (1993).

[68] L. D. Tzuang, K. Fang, P. Nussenzveig, S. Fan, and M. Lipson, Non-reciprocal phase shift induced by an effective magnetic flux for light, Nat. Photon. 8, 701 (2014).

[69] M. G. Clerc, M. A. Ferré, S. Coulibaly, R. G. Rojas, and M. Tlidi, Chimera-like states in an array of coupled-waveguide resonators, Opt. Lett. 42, 2906 (2017).

[70] D. Agafontsev and V. E. Zakharov, Integrable turbulence and formation of rogue waves, Nonlinearity 28, 2791 (2015).

[71] A. E. Kraych, D. Agafontsev, S. Randoux, and P. Suret, Statistical Properties of the Nonlinear Stage of Modulation Instability in Fiber Optics, Phys. Rev. Lett. 123, 093902 (2019).

[72] Y. K. Chembo and C. R. Menyuk, Spatiotemporal LugiatoLefever formalism for Kerr-comb generation in whisperinggallery-mode resonators, Phys. Rev. A 87, 053852 (2013). 